\title{
p-wave superfluidity in mixtures of ultracold Fermi and spinor Bose gases
}

\author{
O. I. Matsyshyn, ${ }^{1}$ A. I. Yakimenko, ${ }^{1}$ E. V. Gorbar,,${ }^{1,2}$ S. I. Vilchinskii, ${ }^{1}$ and V. V. Cheianov ${ }^{3}$ \\ ${ }^{1}$ Department of Physics, Taras Shevchenko National University of Kyiv, 64/13 Volodymyrska Street, Kyiv 01601, Ukraine \\ ${ }^{2}$ Bogolyubov Institute for Theoretical Physics, 14-b Metrologichna, Kyiv 03680, Ukraine \\ ${ }^{3}$ Instituut-Lorentz, Universiteit Leiden, P.O. Box 9506, 2300 RA Leiden, The Netherlands
}

(Received 13 April 2018; published 17 October 2018)

\begin{abstract}
We reveal that the $p$-wave superfluid can be realized in a mixture of fermionic and $F=1$ bosonic gases. We derive a general set of the gap equations for gaps in the $s$ and $p$ channels. It is found that the spin-spin Bose-Fermi interactions favor the $p$-wave pairing and naturally suppress the pairing in the $s$ channel. The gap equations for the polar phase of $p$-wave superfluid fermions are numerically solved. Our study suggests a way to realize a pure $p$-wave superfluid in a well-controlled environment of atomic physics.
\end{abstract}

DOI: 10.1103/PhysRevA.98.043620

\section{INTRODUCTION}

Experimental realisation of a fermionic $p$-wave superfluid is one of the important challenges in ultracold atomic physics. If achieved in laboratory, it would mark an important milestone in the development of hardware for fault-tolerant quantum state manipulation [1]. Indeed, vortex excitations in a two-dimensional chiral version of such a condensate have been predicted [2] to exhibit non-Abelian braiding, which is the core underlying principle of topologically protected quantum computation [3]. A few systems are already known today, which are believed to exhibit chiral $p$-wave pairing. These include the $v=5 / 2$ incompressible quantum Hall fluid [4], superfluid ${ }^{3} \mathrm{He}$ at high pressure [5], strontium ruthenate [6], and superconductor-topological insulator hybrid structures [7]. These systems, however, are not well suited for the experimental realization of quasiparticle braiding, which is mainly due to a limited local control of their parameters. Much hope is, therefore, devoted to the much better controlled ultracold atomic gases.

Theoretical proposals for $p$-wave superfluidity in a ultracold fermionic gas suggested to use Feshbach resonances in order to tune the $p$-wave collision channel [8]. Unfortunately, such resonant condensates were found to be extremely short lived due to the three-body recombination and other decay mechanisms [8-11]. Further theoretical work explored alternative mechanisms of pairing, which would be free of the disadvantages of Feshbach resonances. A number of interesting proposals have emerged such as microwave dressed polar molecules [12], synthetic spin-orbit coupling [13-16], the quantum Zeno effect [17], and driven dissipation [18]. One important group of proposals rests on a traditional view that the nature of fermionic pairing depends on the nature of the agent, which mediates the attraction between fermions. In ultracold atomic systems, it is natural to choose a Bose-Einstein condensate (BEC) as such a mediator. The possibility of a Cooper pairing in the $p$ wave channel in a fermion-boson mixture was originally discussed in Ref. [19], albeit in a spin-polarized system. More recent work explored pairing in a Fermi gas embedded in a spinless Bose condensate [20-22]. In two recent papers $[23,24]$, it was proposed that a fermion-boson system in mixed dimensions may exhibit an enhanced $p$-wave pairing.

One of the most intriguing properties of the $p$-wave paired fermion superfluid is its ability to host half-quantum vortices which have bind unpaired Majorana modes obeying non-Abelian braiding statistics. Half-quantum vortices were theoretically predicted in the 1970s in the context of studies of liquid ${ }^{3} \mathrm{He}-\mathrm{A}$; however, their experimental observation was impeded by various stability issues arising, in particular, from dipole and spin-orbit interactions [25-27]. It was only in 2016 that the first observation of a half-quantum vortex in superfluid ${ }^{3} \mathrm{He}$ in naftene matrix was eventually reported [28]. Around the same time, stable half-quantum vortices were observed in a polar Bose-Einstein condensate [29]; however, such vortices do not possess Majorana zero modes on their own. In this work, we find that using a polar BEC as the medium inducing $p$-wave pairing between the fermions, the direction of the quantization axis of the polar condensate determines the spin state of the $p$-wave superfluid. Thus, a stable half-quantum vortex in the polar BEC host automatically induces a dynamically stable half-quantum vortex in the $p$-wave paired fluid. This makes the present setup an interesting alternative to the fully polarized fermionic fluid considered in Ref. [19].

In this paper, we explore a fermion-boson mixture in which the bosons form a polar spinor condensate. We derive the effective action for the fermions and find that in addition to the usual BCS-type attraction, the Bose condensate excitations mediate a different type of interaction which takes the form of a spin-dependent X-Y exchange. We demonstrate that such an interaction between the fermions may suppress pairing in the $s$-wave channel while favoring the $p$-wave pairing channel. For a particular $p$-wave phase, we derive and solve the complete set of the Eliashberg equations determining the gap parameter and explore the dependence of the gap parameter on the tunable parameters of the mixture.

\section{MODEL}

Let us describe our model of a three-dimensional (3D) spatially homogeneous mixture of electrically neutral bosonic atoms with hyperspin $F=1$ and spin- $\frac{1}{2}$ fermionic atoms. We 
assume that an external magnetic field is absent. The second quantized Hamiltonian of the mixture of dilute Fermi and spinor Bose gases reads

$$
\hat{H}=\int\left[\hat{\mathcal{H}}_{B}(\mathbf{r})+\hat{\mathcal{H}}_{F}(\mathbf{r})+\hat{\mathcal{H}}_{B F}(\mathbf{r})\right] d \mathbf{r} .
$$

The Hamiltonian density $\hat{\mathcal{H}}_{B}(\mathbf{r})$ describes interacting $F=1$ spinor bosonic atoms [30-33],

$$
\hat{\mathcal{H}}_{B}=-\frac{\hbar^{2}}{2 M_{B}} \sum_{m} \hat{\Psi}_{m}^{\dagger} \nabla^{2} \hat{\Psi}_{m}+\frac{1}{2} c_{0}: \hat{n}_{B}^{2}:+\frac{1}{2} c_{2}: \hat{\mathbf{F}}^{2}:,
$$

where the field operators $\hat{\Psi}(\mathbf{r})$ obey the bosonic commutation relations

$$
\left[\hat{\Psi}_{m}(\mathbf{r}), \hat{\Psi}_{m^{\prime}}^{\dagger}\left(\mathbf{r}^{\prime}\right)\right]=\delta_{m, m^{\prime}} \delta\left(\mathbf{r}-\mathbf{r}^{\prime}\right)
$$

and : $\hat{A}:$ denotes normally ordered operator $\hat{A}$. The $j$ th component of the vector spin density operator for bosons $\hat{\mathbf{F}}$ equals

$$
\hat{\mathbf{F}}_{j}=\sum_{m, m^{\prime}} \hat{\Psi}_{m}^{\dagger}(\mathbf{r})\left\{F_{j}\right\}_{m, m^{\prime}} \hat{\Psi}_{m^{\prime}}(\mathbf{r}),
$$

where $F_{j}$ are the $3 \times 3$ matrices of the vector representation of the rotation group. Further,

$$
c_{0}=\frac{4 \pi \hbar^{2}}{3 M_{B}}\left(a_{B}^{(0)}+2 a_{B}^{(2)}\right), \quad c_{2}=\frac{4 \pi \hbar^{2}}{3 M_{B}}\left(a_{B}^{(2)}-a_{B}^{(0)}\right)
$$

are symmetric and spin-dependent interaction constants, respectively, and $a_{B}^{(J)}$ is the scattering length in the state with spin $J$. We also introduced the number density $\hat{n}_{B}(\mathbf{r})=$ $\sum_{m} \hat{\Psi}_{m}^{\dagger}(\mathbf{r}) \hat{\Psi}_{m}(\mathbf{r}), m \in\{-1,0,+1\}$, of the bosonic field. It is known that the spin-1 BEC in the absence of an external magnetic field has two phases: ferromagnetic $\left(c_{2}<0\right)$ and polar $\left(c_{2}>0\right)$. We consider in this paper the polar spinor BEC of ${ }^{23} \mathrm{Na}$ atoms with $a_{B}^{(0)}=50.0, a_{B}^{(2)}=55.0$ (see, e.g., [34]) in units of the Bohr radius.

The fermion Hamiltonian density $\hat{\mathcal{H}}_{F}(\mathbf{r})$ is given by

$$
\hat{\mathcal{H}}_{F}(\mathbf{r})=\sum_{\nu} \hat{f}_{v}^{\dagger}\left(-\frac{\hbar^{2}}{2 M_{F}} \nabla^{2}-\mu\right) \hat{f}_{\nu}+\frac{1}{2} g_{F}: \hat{n}_{F}^{2}:
$$

where $\mu$ is the fermion chemical potential, and the fermion field operators $\hat{f}$ obey the anticommutation relations

$$
\left\{\hat{f}_{\nu}(\mathbf{r}), \hat{f}_{v^{\prime}}^{\dagger}\left(\mathbf{r}^{\prime}\right)\right\}=\delta_{v, v^{\prime}} \delta\left(\mathbf{r}-\mathbf{r}^{\prime}\right) .
$$

Further, $\quad \hat{n}_{F}(\mathbf{r})=\sum_{v} \hat{f}_{v}^{\dagger}(\mathbf{r}) \hat{f}_{v}(\mathbf{r}), \quad v \in\{\uparrow, \downarrow\}, \quad g_{F}=$ $4 \pi \hbar^{2} a_{F} / M_{F}$ is the coupling constant of the fermion density-density interaction, and $a_{F}$ is the $s$-wave scattering length. The different canonical ensembles are used for bosons and fermions.

The interactions between bosons and fermions in the leading order in densities and spins are described in Hamiltonian (1) by the term

$$
\hat{\mathcal{H}}_{B F}(\mathbf{r})=\frac{\alpha}{2} \hat{n}_{B} \hat{n}_{F}+\beta \hat{\mathbf{S}} \cdot \hat{\mathbf{F}}
$$

where $\quad \alpha=8 \pi \hbar^{2}\left(2 a_{B F}^{(1 / 2)}+a_{B F}^{(3 / 2)}\right) /\left(3 M_{B F}\right), \quad$ and $\quad \beta=$ $8 \pi \hbar^{2}\left(a_{B F}^{(1 / 2)}-a_{B F}^{(3 / 2)}\right) /\left(3 M_{B F}\right)$ are the density-density and spin-spin Bose-Fermi interactions, respectively, $a_{B F}^{(J)}$ is the scattering length in the state with spin $J$, and
$M_{B F}=2 M_{B} M_{F} /\left(M_{B}+M_{F}\right)$ is the reduced mass for a boson of mass $M_{B}$ and a fermion of mass $M_{F}$.

The spin-density operator for fermions has the standard form

$$
\hat{\mathbf{S}}_{j}=\frac{1}{2} \sum_{v, v^{\prime}} \hat{f}_{v}^{\dagger}(\mathbf{r})\left\{\sigma_{j}\right\}_{v, v^{\prime}} \hat{f}_{v^{\prime}}(\mathbf{r})
$$

where $\hat{\sigma}_{j}$ are the Pauli matrices for spin- $\frac{1}{2}$ fermions.

In what follows, we neglect the back reaction of the fermion sector on the Bose condensate assuming that the Bose-Fermi interactions are weak.

In our study, we consider different values of bosonic density $n_{B}$ of ${ }^{23} \mathrm{Na}$ atoms at the fixed fermionic density $n_{F}=$ $10^{14} \mathrm{~cm}^{-3}$ of ${ }^{40} \mathrm{~K}$ atoms with the Fermi temperature $T_{F}=$ $E_{F} / k_{B}=1248 \mathrm{nK}$, where $E_{F}=\left(3 \pi^{2} n_{F}\right)^{2 / 3} \hbar^{2} /\left(2 M_{F}\right)$ is the Fermi energy.

\section{GAP EQUATIONS AND SOLUTIONS FOR POLAR SUPERFLUID PHASE}

Let us show that the effective fermion-fermion interaction mediated by the interaction with $F=1$ bosons may give rise to the $p$-wave pairing between the fermions. Using Hamiltonian (1), we find that the fermion-boson interactions lead to the following effective fermion action:

$$
S_{\mathrm{eff}}=S_{0}+S_{\mathrm{int}},
$$

where

$$
\begin{aligned}
S_{0}= & \int d \mathbf{r} d t\left[\sum_{v} f_{v}^{\dagger}\left(i \frac{\partial}{\partial t}+\frac{\hbar^{2}}{2 M_{F}} \nabla^{2}+\mu\right) f_{v}\right. \\
& \left.-\frac{1}{2} g_{F}: n_{F}^{2}(\mathbf{r}):\right]
\end{aligned}
$$

is the free fermion action and the interaction term is

$$
\begin{aligned}
S_{\text {int }}= & \sum_{\mathbf{k} \neq 0} \frac{\epsilon_{\mathbf{k}} n_{B}}{2} \int d \omega\left\{\frac { \beta ^ { 2 } } { E _ { \mathbf { k } , 1 } ^ { 2 } - ( \hbar \omega ) ^ { 2 } } \left[\sigma_{+}(k) \sigma_{-}(-k)\right.\right. \\
& \left.+\sigma_{-}(k) \sigma_{+}(-k)\right] \\
& \left.+\frac{\alpha^{2}}{2\left[E_{\mathbf{k}, 0}^{2}-(\hbar \omega)^{2}\right]} n_{F}(k) n_{F}(-k)\right\} .
\end{aligned}
$$

Here, $\epsilon_{\mathbf{k}}=\hbar^{2} \mathbf{k}^{2} /\left(2 M_{B}\right), k=(\omega, \mathbf{k}), r=(t, \mathbf{r}), \Omega$ is the system's volume,

$$
\sigma_{ \pm}(k)=\frac{1}{2 \sqrt{\Omega}} \int e^{i k r} f^{\dagger}(r)\left(\sigma_{x} \pm i \sigma_{y}\right) f(r) d r,
$$

and

$$
\begin{aligned}
& E_{\mathbf{k}, 0}=\sqrt{\epsilon_{\mathbf{k}}\left(\epsilon_{\mathbf{k}}+2 c_{0} n_{B}\right)}, \\
& E_{\mathbf{k}, \pm 1}=\sqrt{\epsilon_{\mathbf{k}}\left(\epsilon_{\mathbf{k}}+2 c_{2} n_{B}\right)}
\end{aligned}
$$

are the Bogoliubov dispersion relations of the Bose gas excitations (see, e.g., [30]).

Before proceeding to derivation and a detailed analysis of gap equations, it is instructive to consider a mean-field 
Hamiltonian for the $s$-wave pairing state,

$$
H_{\mathrm{int}} \sim-\int d \mathbf{r}\left\{G_{n} \operatorname{Tr}\left[\Delta^{+} \Delta\right]+G_{s} \sum_{l=+,-} \operatorname{Tr}\left[\sigma_{l} \Delta^{+} \sigma_{l} \Delta\right]\right\},
$$

where $\Delta$ is the pairing gap, and $G_{n}$ and $G_{s}$ are constants of density-density and spin-spin interactions.

Using definition (8) of the operators $\sigma_{ \pm}$, it is straightforward to show that the contributions of density-density and spin-spin interactions appear with opposite signs in the $s$-wave channel. Thus, while density-density interactions cause an effective attraction, spin-spin interactions lead to an effective repulsion in the $s$-wave channel. The further rigorous analysis not only confirms this preliminary conclusion, but also reveals that spin-mediated interactions drive an additional attraction in the $p$-wave channel.

Using the effective action (5), we find the following Dyson's equations in the Matsubara formalism for the fermion Green function $G\left(\mathbf{k}, \omega_{n}\right)$ and the anomalous Gor'kov function $F\left(\mathbf{k}, \omega_{n}\right)$ :

$$
\begin{aligned}
& \left(i \hbar \omega_{n}-\xi_{\mathbf{k}}\right) G\left(\mathbf{k}, \omega_{n}\right)+\Delta\left(\mathbf{k}, \omega_{n}\right) F^{\dagger}\left(\mathbf{k}, \omega_{n}\right)=\hbar, \\
& \left(i \hbar \omega_{n}+\xi_{\mathbf{k}}\right) F^{\dagger}\left(\mathbf{k}, \omega_{n}\right)+\Delta^{\dagger}\left(\mathbf{k}, \omega_{n}\right) G\left(\mathbf{k}, \omega_{n}\right)=0,
\end{aligned}
$$

where $\xi_{k}=\hbar^{2} \mathbf{k}^{2} /\left(2 M_{F}\right)-\mu$ and $\omega_{n}=k_{B} T(2 n+1) \pi / \hbar$ is the Matsubara fermion frequency. In addition, neglecting the retardation effects, the gap function $\Delta\left(\mathbf{k}, \omega_{n}\right)$ is expressed through the Gor'kov function as follows:

$$
\begin{aligned}
\Delta(\mathbf{k})= & -\frac{k_{B} T}{\hbar} \sum_{n^{\prime}, \mathbf{k}^{\prime} \neq 0}\left[\frac{g_{F}}{\Omega} F\left(\mathbf{k}-\mathbf{k}^{\prime}, \omega_{n^{\prime}}\right)\right. \\
& -\tilde{g}_{n}\left(\mathbf{k}^{\prime}, \omega_{n^{\prime}}\right) F\left(\mathbf{k}-\mathbf{k}^{\prime}, \omega_{n}-\omega_{n^{\prime}}\right) \\
& \left.-\tilde{g}_{s}\left(\mathbf{k}^{\prime}, \omega_{n^{\prime}}\right) \sum_{l=+,-} \sigma_{l} F\left(\mathbf{k}-\mathbf{k}^{\prime}, \omega_{n}-\omega_{n^{\prime}}\right) \sigma_{l}\right],
\end{aligned}
$$

where

$$
\begin{aligned}
& \tilde{g}_{n}\left(\mathbf{k}, \omega_{n^{\prime}}\right)=\frac{c_{n}}{E_{\mathbf{k}, 0}^{2}+\left(\hbar \omega_{n^{\prime}}\right)^{2}}, \quad c_{n}=\frac{\alpha^{2} n_{B} \epsilon_{\mathbf{k}}}{2 \Omega}, \\
& \tilde{g}_{s}\left(\mathbf{k}, \omega_{n^{\prime}}\right)=\frac{c_{s}}{E_{\mathbf{k}, 1}^{2}+\left(\hbar \omega_{n^{\prime}}\right)^{2}}, \quad c_{s}=\frac{\beta^{2} n_{B} \epsilon_{\mathbf{k}}}{\Omega} .
\end{aligned}
$$

An ansatz for the pairing gap, which accounts for both $s$ and $p$-wave channels, is given by

$$
\Delta_{\alpha \beta}(\mathbf{k})=\Delta_{p}\left(i \sigma \sigma_{y}\right)_{\alpha \beta} \mathbf{d}(\mathbf{k})+\Delta_{s}\left(i \sigma_{y}\right)_{\alpha \beta},
$$

where $\Delta_{p}$ and $\Delta_{s}$ are real functions of $|\mathbf{k}|$. As is well known, the order parameter of the $p$-wave phase is proportional to vector $\mathbf{d}$ whose form and symmetries determine the superfluid state.

It is straightforward now to obtain the set of gap equations for $\Delta_{s}$ and $\Delta_{p}$ using the standard procedure (see the Appendix). There are, in principle, many solutions to the gap equations. We follow in our analysis the well-known classification of the ${ }^{3} \mathrm{He}$ phases [35] defined by the form of vector $\mathbf{d}$. In the present work, we restrict our consideration to the polar phase with $\mathbf{d} \sim\left(0,0, k_{z}\right)$, which corresponds to one of the simplest solutions of the gap equations. As shown above, the $s$-wave superfluidity is suppressed by the
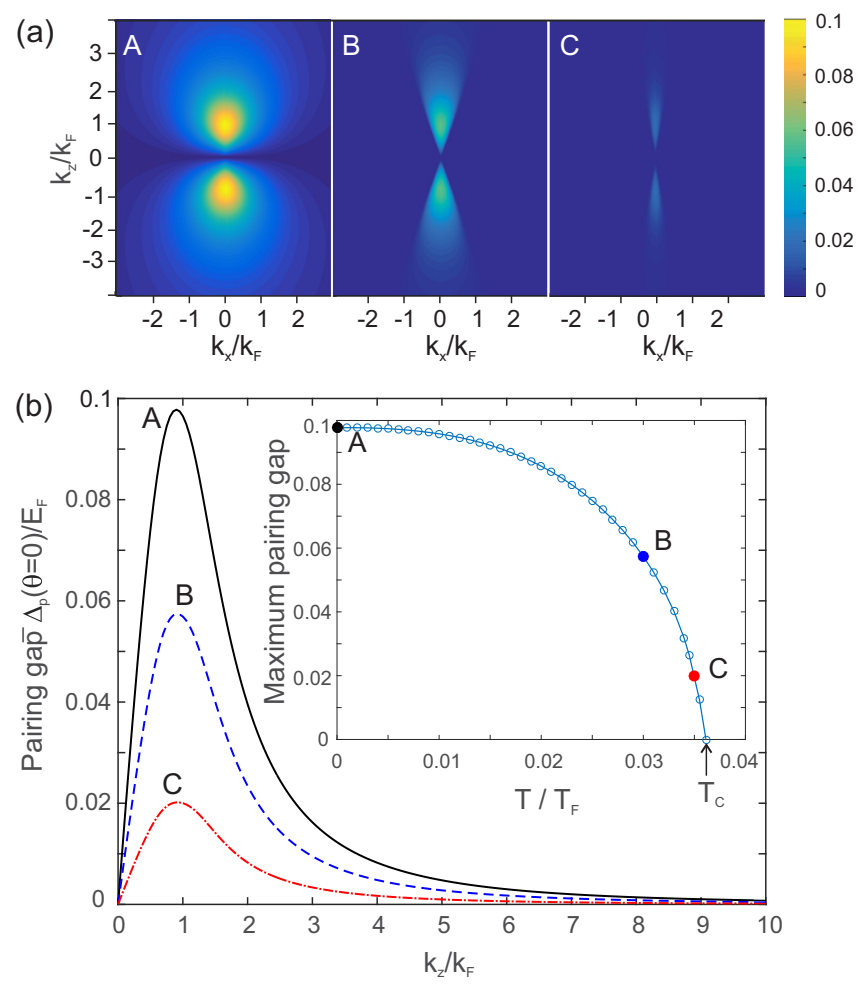

FIG. 1. Typical examples of the pairing gap $\Delta=\Delta_{p} d_{z}$ in the $p$-wave polar phase found numerically at different temperatures (A: $T=0 ; \mathrm{B}: T=0.03 T_{F}$; and $\mathrm{C}: T=0.035 T_{F}$ ) for the interaction constants $\alpha=\beta=0.3 c_{0}, n_{B}=n_{F}=10^{14} \mathrm{~cm}^{-3}$. (a) The cross section of the pairing gap $\left|\Delta\left(k_{x}, k_{y}=0, k_{z}\right)\right|$. (b) The profile of the pairing gap along the $z$ axis. The inset presents the maximum of the pairing gap as a function of temperature. Note that the pairing gap vanishes at the critical temperature $T_{c}$.

spin-induced interactions; thus, we assume for simplicity that $\Delta_{s}=0$ and $\Delta_{p} \neq 0$. Then the gap equation in the polar phase reads

$$
\begin{aligned}
\Delta(\mathbf{k})= & \sum_{\mathbf{k}^{\prime} \neq 0}\left\{c_{n}\left(\frac{W_{0}}{2 E} \tanh \frac{E}{2 k_{B} T}+\frac{Z_{0}}{2 E_{\mathbf{k}, 0}} \tanh \frac{E_{\mathbf{k}, 0}}{2 k_{B} T}\right)\right. \\
& \left.+c_{s}\left(\frac{W_{1}}{2 E} \tanh \frac{E}{2 k_{B} T}+\frac{Z_{1}}{2 E_{\mathbf{k}, 1}} \tanh \frac{E_{\mathbf{k}, 1}}{2 k_{B} T}\right)\right\} \\
& \times \Delta\left(\mathbf{k}-\mathbf{k}^{\prime}\right),
\end{aligned}
$$

where $\Delta(\mathbf{k})=\left|d_{z}\right| \Delta_{p}(|\mathbf{k}|)$,

$$
\begin{aligned}
& W_{i}=\frac{E_{\mathbf{k}, i}^{2}-E^{2}+\hbar^{2} \omega_{0}^{2}}{\left(E_{\mathbf{k}, i}^{2}-E^{2}\right)^{2}+2 \hbar^{2} \omega_{0}^{2}\left(E_{\mathbf{k}, i}^{2}+E^{2}+\frac{\hbar^{2} \omega_{0}^{2}}{2}\right)}, \\
& Z_{i}=\frac{E^{2}-E_{\mathbf{k}, i}^{2}+\hbar^{2} \omega_{0}^{2}}{\left(E_{\mathbf{k}, i}^{2}-E^{2}\right)^{2}+2 \hbar^{2} \omega_{0}^{2}\left(E_{\mathbf{k}, i}^{2}+E^{2}+\frac{\hbar^{2} \omega_{0}^{2}}{2}\right)},
\end{aligned}
$$

with $i=0,1, \omega_{0}=k_{B} T \pi / \hbar$, and $E=\sqrt{\xi_{\mathbf{k}}+\Delta^{2}}$.

Let us discuss the numerical solutions of Eq. (15) obtained by using a stabilized iterative procedure [36]. Since the function $\Delta$ has an axial symmetry connected with rotations around the $z$ axis, it suffices to consider a cross section of its pairing gap at $k_{y}=0$. Figure 1 presents typical examples of this cross 

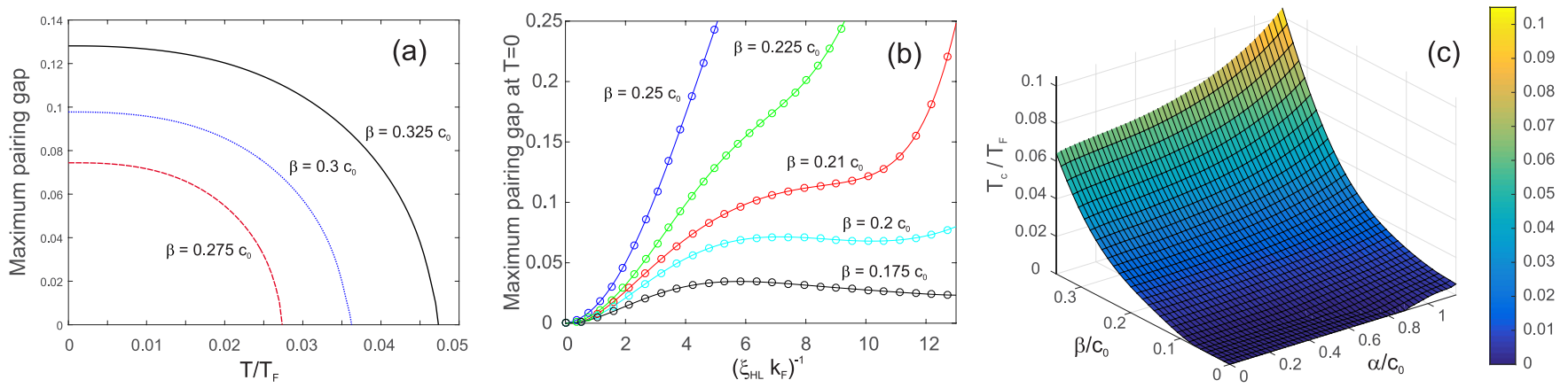

FIG. 2. (a) The maximal pairing gap as a function of temperature for $\alpha=0.3 c_{0}$ and different values of $\beta$ indicated near the curves. (b) The maximal pairing gap at $T=0$ as a function of $\left(k_{F} \xi_{\mathrm{HL}}\right)^{-1}$ at fixed $\alpha=0.3 c_{0}$ and $n_{F}=10^{14} \mathrm{~cm}^{-3}$ plotted for different values of $\beta$. (c) The critical temperature vs the interaction constants $\alpha$ and $\beta$ connected with $c_{0}$. The concentrations of bosons and fermions in (a) and (c) are fixed at $n_{B}=n_{F}=10^{14} \mathrm{~cm}^{-3}$.

section at fixed temperature and interaction constants $\alpha$ and $\beta$. It is seen from Fig. 1 that the maximum of the pairing gap is localized as expected near the points $\left(0,0, \pm k_{F}\right)$ and the maximal pairing gap monotonically decreases as temperature increases [see the inset in Figs. 1(b) and 2(a)]. The pairing gap in the $p$ phase vanishes at some critical temperature $T_{c}$. We investigated the dependence of the critical temperature $T_{c}$ on the ratios $\alpha / c_{0}$ and $\beta / c_{0}$ of boson-fermion interactions to the strength of the boson-boson interaction $c_{0}$ [see Fig. 2(c)]. According to Fig. 2(c), the critical temperature $T_{c}$ can be significantly increased through an interplay of the density-density and spin-spin interactions between fermions and spinor BEC.

The pairing gap is a function of interaction constants $\alpha, \beta, c_{0}, c_{2}$ and densities of the Bose and Fermi gases. Figure 2(b) illustrates how the pairing gap is affected by variation of the Bose-gas density. The nontrivial dependence of the maximal gap $\Delta_{p}$ on $\left(k_{F} \xi_{\mathrm{HL}}\right)^{-1}$, where $\xi_{\mathrm{HL}}=$ $1 / \sqrt{8 \pi n_{B}\left(a_{B}^{(0)}+2 a_{B}^{(2)}\right) / 3}$, at $T=0$ comes from the Bogoliubov dispersion relations (9) and (10) of the Bose gas. Such a dependence should be taken into account in the experimental observation of the $p$-wave superfluid state.

\section{CONCLUSIONS}

We showed that a mixture of dilute ultracold $F=1$ spinor atomic BEC and fermionic atoms provides a promising platform for the realization of the $p$-wave superfluid state in three dimensions. Such a mixture makes it possible to avoid the problem of three-body inelastic collisional loss in a singlecomponent ultracold Fermi gas, where the Feshbach resonance is used to enhance the $p$-wave cross section to values larger than the $s$-wave cross section. We derived a general set of the gap equations for the pairing gaps in the $s$ and $p$ channels. It is found that the spin-induced interactions in a mixture of $F=1$ spinor atomic BEC and fermionic atoms naturally suppress the $s$-wave pairing. At the same time, the $p$ wave pairing is driven not only by density-induced interaction, but also by spin-induced interactions and it emerges even for repulsive interaction between fermions.

We studied the simplest case of the $p$-wave triplet pairing defined by the polar phase with $\mathbf{d}=\left(0,0, k_{z}\right)$. For the case of dilute Fermi ${ }^{40} \mathrm{~K}$ and Bose ${ }^{23} \mathrm{Na}$ atomic gases, we found numerically the solution to the gap equations of the polar phase with the vanishing $s$-wave order parameter $\Delta_{s}=0$. While the dependence of the maximal value of the gap on temperature is a monotonically decreasing function, its dependence on the density of bosons $n_{B}$ is nonmonotonous for sufficiently small values of the spin-spin Bose-Fermi interaction $\beta$. Such a dependence comes from the corresponding nontrivial dependence of the Bogoliubov dispersion relations on $n_{B}$.

We hope that the results presented in this paper will stimulate experiments to observe the $p$-wave superfluid in a mixture of ultracold Fermi and spinor Bose gases. Moreover, the study of the $p$-wave superfluidity in a well-controlled environment of atomic physics could help to elucidate the properties of topologically nontrivial superfluids.

\section{ACKNOWLEDGMENTS}

O.M. and A.Y. acknowledge the support of the Project No. 1/30-2015 "Dynamics and topological structures in BoseEinstein condensates of ultracold gases" of the KNU Branch Target Training at the NAS of Ukraine. A.Y. acknowledges the hospitality of the Leiden University, where this work was commenced. The work of E.V.G. was partially supported by the Program of Fundamental Research of the Physics and Astronomy Division of the National Academy of Sciences of Ukraine.

\section{APPENDIX: GENERAL GAP EQUATIONS}

Using ansatz (14), we rewrite Eqs. (11) and (12) as

$$
G_{\alpha \beta}\left(\mathbf{k}, \omega_{n}\right)=-\hbar\left(i \hbar \omega_{n}+\xi_{\mathbf{k}}\right) \frac{\left(\hbar^{2} \omega_{n}^{2}+\xi_{\mathbf{k}}^{2}+\Delta^{2}\right) \delta_{\alpha \beta}-\Delta^{2} \mathbf{M} \boldsymbol{\sigma}_{\alpha \beta}}{\left(\hbar^{2} \omega_{n}^{2}+E_{+}^{2}\right)\left(\hbar^{2} \omega_{n}^{2}+E_{-}^{2}\right)}
$$




$$
\begin{aligned}
F_{\alpha \beta}\left(\mathbf{k}, \omega_{n}\right)= & \hbar \Delta_{p}\left(\boldsymbol{\sigma} i \sigma_{y}\right)_{\alpha \beta} \frac{\left(\hbar^{2} \omega_{n}^{2}+\xi_{\mathbf{k}}^{2}+\Delta^{2}\right) \mathbf{d}-i \Delta^{2}[\mathbf{M} \times \mathbf{d}]}{\left(\hbar^{2} \omega_{n}^{2}+E_{+}^{2}\right)\left(\hbar^{2} \omega_{n}^{2}+E_{-}^{2}\right)}+\hbar \Delta_{s} \frac{\left\{\hbar^{2} \omega_{n}^{2}+\xi_{\mathbf{k}}^{2}+\Delta^{2}-2 \Delta_{p}^{2}(\operatorname{Re}[\mathbf{d}] \cdot \mathbf{d})\right\} i \sigma_{y, \alpha \beta}}{\left(\hbar^{2} \omega_{n}^{2}+E_{+}^{2}\right)\left(\hbar^{2} \omega_{n}^{2}+E_{-}^{2}\right)} \\
& +\hbar \Delta_{s} \frac{\Delta^{2}\left(\sigma i \sigma_{y}\right)_{\alpha \beta} \mathbf{M}}{\left(\hbar^{2} \omega_{n}^{2}+E_{+}^{2}\right)\left(\hbar^{2} \omega_{n}^{2}+E_{-}^{2}\right)}
\end{aligned}
$$

where

$$
\begin{aligned}
\Delta^{2} & =\Delta_{p}^{2}|\mathbf{d}|^{2}+\Delta_{s}^{2}, \\
\mathbf{m}(\mathbf{k}) & =i\left[\mathbf{d}(\mathbf{k}) \times \mathbf{d}^{*}(\mathbf{k})\right], \\
\mathbf{M} & =\frac{\Delta_{p}^{2} \mathbf{m}+2 \Delta_{p} \Delta_{s} \operatorname{Re}[\mathbf{d}]}{\Delta^{2}} \\
E_{ \pm} & =\sqrt{\xi_{\mathbf{k}}^{2}+\Delta^{2}(1 \pm|M|)}
\end{aligned}
$$

The general gap equations read

$$
\begin{gathered}
d_{x}(\mathbf{k}) \Delta_{p}\left(\mathbf{k}, \omega_{k}\right)=k_{B} T \sum_{n, \mathbf{k}^{\prime} \neq 0} \tilde{g}_{n}\left(\mathbf{k}^{\prime}, \omega_{k}-\omega_{n}\right) \Delta_{p}\left(\mathbf{k}-\mathbf{k}^{\prime}, \omega_{n}\right) v_{x}\left(\mathbf{k}-\mathbf{k}^{\prime}, \omega_{n}\right), \\
d_{y}(\mathbf{k}) \Delta_{p}\left(\mathbf{k}, \omega_{k}\right)=k_{B} T \sum_{n, \mathbf{k}^{\prime} \neq 0} \tilde{g}_{n}\left(\mathbf{k}^{\prime}, \omega_{k}-\omega_{n}\right) \Delta_{p}\left(\mathbf{k}-\mathbf{k}^{\prime}, \omega_{n}\right) v_{y}\left(\mathbf{k}-\mathbf{k}^{\prime}, \omega_{n}\right), \\
d_{z}(\mathbf{k}) \Delta_{p}\left(\mathbf{k}, \omega_{k}\right)=k_{B} T \sum_{n, \mathbf{k}^{\prime} \neq 0}\left[\tilde{g}_{n}\left(\mathbf{k}^{\prime}, \omega_{k}-\omega_{n}\right)+\tilde{g}_{s}\left(\mathbf{k}^{\prime}, \omega_{k}-\omega_{n}\right)\right] \Delta_{p}\left(\mathbf{k}-\mathbf{k}^{\prime}, \omega_{n}\right) v_{z}\left(\mathbf{k}-\mathbf{k}^{\prime}, \omega_{n}\right), \\
\Delta_{s}\left(\mathbf{k}, \omega_{k}\right)=k_{B} T \sum_{n, \mathbf{k}^{\prime} \neq 0}\left[-\frac{g_{F}}{\Omega}+\tilde{g}_{n}\left(\mathbf{k}^{\prime}, \omega_{k}-\omega_{n}\right)-\tilde{g}_{s}\left(\mathbf{k}^{\prime}, \omega_{k}-\omega_{n}\right)\right] \Delta_{s}\left(\mathbf{k}-\mathbf{k}^{\prime}, \omega_{n}\right) \varepsilon\left(\mathbf{k}-\mathbf{k}^{\prime}, \omega_{n}\right),
\end{gathered}
$$

where

$$
\begin{aligned}
& \boldsymbol{v}\left(\mathbf{k}, \Delta_{p}, \Delta_{s}\right)= \frac{1}{\left[\hbar^{2} \omega_{n}^{2}+E_{+}^{2}(\mathbf{k})\right]\left[\hbar^{2} \omega_{n}^{2}+E_{-}^{2}(\mathbf{k})\right]}\left\{\left[\hbar^{2} \omega_{n}^{2}+\xi_{\mathbf{k}}^{2}+\Delta^{2}(\mathbf{k})\right] \mathbf{d}(\mathbf{k})-i \Delta_{p}^{2}(\mathbf{k})[\mathbf{m}(\mathbf{k}) \times \mathbf{d}(\mathbf{k})]\right. \\
&\left.+2 \Delta_{s}(\mathbf{k}) \Delta_{p}(\mathbf{k}) \mathbf{m}(\mathbf{k})+2 \Delta_{s}^{2}(\mathbf{k}) \operatorname{Re}[\mathbf{d}(\mathbf{k})]\right\}, \\
& \varepsilon\left(\mathbf{k}, \Delta_{p}, \Delta_{s}\right)= \frac{1}{\left[\hbar^{2} \omega_{n}^{2}+E_{+}^{2}(\mathbf{k})\right]\left[\hbar^{2} \omega_{n}^{2}+E_{-}^{2}(\mathbf{k})\right]}\left[\hbar^{2} \omega_{n}^{2}+\xi_{\mathbf{k}}^{2}+\Delta^{2}(\mathbf{k})-2 \Delta_{p}^{2}(\mathbf{k})\{\operatorname{Re}[\mathbf{d}(\mathbf{k})] \cdot \mathbf{d}(\mathbf{k})\}\right], \\
& \Delta^{2}(\mathbf{k})=\Delta_{p}^{2}(\mathbf{k})|d(\mathbf{k})|^{2}+\Delta_{s}^{2}(\mathbf{k}), \\
& E_{ \pm}(\mathbf{k})=\sqrt{\xi_{\mathbf{k}}^{2}+\Delta^{2}(\mathbf{k}) \pm \Delta_{p}^{2}(\mathbf{k}) \mid \mathbf{m}(\mathbf{k})+2 \frac{\Delta_{s}(\mathbf{k})}{\Delta_{p}(\mathbf{k})} \operatorname{Re}[\mathbf{d}(\mathbf{k})]} .
\end{aligned}
$$

It is not surprising that the density-density Bose-Fermi interaction produces effective attractive interactions in the $s$-wave channel. It is particularly remarkable that the spin-spin Bose-Fermi interactions lead to an effective repulsive interaction in the $s$-wave channel. Thus, in sharp contrast with the $s$-wave superfluidity, for the $p$-wave superfluidity, both the spin-spin and the density-density Bose-Fermi interactions lead to an effective attraction that results in the realization of a $p$-wave superfluid.

[1] C. Nayak, S. H. Simon, A. Stern, M. Freedman, and S. Das Sarma, Rev. Mod. Phys. 80, 1083 (2008).

[2] D. A. Ivanov, Phys. Rev. Lett. 86, 268 (2001).

[3] A. Y. Kitaev, Ann. Phys. 303, 2 (2003).

[4] G. Moore and N. Read, Nucl. Phys. B 360, 362 (1991).

[5] G. E. Volovik, The Universe in a Helium Droplet, Vol. 117 (Oxford University Press, Oxford, 2003).

[6] C. Kallin, Rep. Prog. Phys. 75, 042501 (2012).
[7] S. Charpentier, L. Galletti, G. Kunakova, R. Arpaia, Y. Song, R. Baghdadi, S. M. Wang, A. Kalaboukhov, E. Olsson, F. Tafuri et al., Nat. Commun. 8, 2019 (2017).

[8] J. Levinsen, N. R. Cooper, and V. Gurarie, Phys. Rev. A 78, 063616 (2008).

[9] J. Zhang, E. G. M. van Kempen, T. Bourdel, L. Khaykovich, J. Cubizolles, F. Chevy, M. Teichmann, L. Tarruell, S. J. J. M. F. Kokkelmans, and C. Salomon, Phys. Rev. A 70, 030702 (2004). 
[10] J. P. Gaebler, J. T. Stewart, J. L. Bohn, and D. S. Jin, Phys. Rev. Lett. 98, 200403 (2007).

[11] M. Jona-Lasinio, L. Pricoupenko, and Y. Castin, Phys. Rev. A 77, 043611 (2008).

[12] N. R. Cooper and G. V. Shlyapnikov, Phys. Rev. Lett. 103, 155302 (2009).

[13] C. Zhang, S. Tewari, R. M. Lutchyn, and S. Das Sarma, Phys. Rev. Lett. 101, 160401 (2008).

[14] M. Sato, Y. Takahashi, and S. Fujimoto, Phys. Rev. Lett. 103, 020401 (2009).

[15] R. A. Williams, L. J. LeBlanc, K. Jimenez-Garcia, M. C. Beeler, A. R. Perry, W. D. Phillips, and I. B. Spielman, Science 335, 314 (2012).

[16] B. Juliá-Díaz, T. Graß, O. Dutta, D. Chang, and M. Lewenstein, Nat. Commun. 4, 2046 (2013).

[17] Y.-J. Han, Y.-H. Chan, W. Yi, A. J. Daley, S. Diehl, P. Zoller, and L.-M. Duan, Phys. Rev. Lett. 103, 070404 (2009).

[18] C.-E. Bardyn, M. A. Baranov, E. Rico, A. İmamoğlu, P. Zoller, and S. Diehl, Phys. Rev. Lett. 109, 130402 (2012).

[19] D. V. Efremov and L. Viverit, Phys. Rev. B 65, 134519 (2002).

[20] D.-W. Wang, M. D. Lukin, and E. Demler, Phys. Rev. A 72, 051604 (2005)

[21] R. Onofrio, Physics Uspekhi 59, 1129 (2016).

[22] P. Massignan, A. Sanpera, and M. Lewenstein, Phys. Rev. A 81, 031607 (2010)

[23] Z. Wu and G. M. Bruun, Phys. Rev. Lett. 117, 245302 (2016).
[24] L. Mathey, S.-W. Tsai, and A. H. Castro Neto, Phys. Rev. Lett. 97, 030601 (2006).

[25] P. J. Hakonen, K. K. Nummila, J. T. Simola, L. Skrbek, and G. Mamniashvili, Phys. Rev. Lett. 58, 678 (1987).

[26] M. Yamashita, K. Izumina, A. Matsubara, Y. Sasaki, O. Ishikawa, T. Takagi, M. Kubota, and T. Mizusaki, Phys. Rev. Lett. 101, 025302 (2008).

[27] T. Kawakami, Y. Tsutsumi, and K. Machida, Phys. Rev. B 79, 092506 (2009).

[28] S. Autti, V. V. Dmitriev, J. T. Mäkinen, A. A. Soldatov, G. E. Volovik, A. N. Yudin, V. V. Zavjalov, and V. B. Eltsov, Phys. Rev. Lett. 117, 255301 (2016).

[29] S. W. Seo, S. Kang, W. J. Kwon, and Y.-i. Shin, Phys. Rev. Lett. 115, 015301 (2015).

[30] T.-L. Ho, Phys. Rev. Lett. 81, 742 (1998).

[31] T. Ohmi and K. Machida, J. Phys. Soc. Jpn. 67, 1822 (1998).

[32] Y. Kawaguchi and M. Ueda, Phys. Rep. 520, 253 (2012).

[33] D. M. Stamper-Kurn and M. Ueda, Rev. Mod. Phys. 85, 1191 (2013).

[34] L. Pitaevskii and S. Stringari, Bose-Einstein Condensation and Superfluidity (Oxford University Press, Oxford, 2016).

[35] V. Mineev and K. Samokhin, Introduction to the Theory of Unusual Superconductivity (CRC, Boca Raton, FL, 1999).

[36] V. Petviashvili and V. Yan'kov, in Reviews in Plasma Physics, edited by B. B. Kadomtsev (Consultants Bureau, New York, 1989). 